\title{
MicroRNA-205 suppresses the invasion and epithelial-mesenchymal transition of human gastric cancer cells
}

\author{
CHANG XU ${ }^{1 *}$, MING'E LI $^{2 *}$, LIN ZHANG $^{3}$, YUEYANG BI $^{4}$, PEIYUAN WANG $^{3}, \mathrm{JUN} \mathrm{LI}^{3}$ and XINGYUE JIANG \\ ${ }^{1}$ Department of Radiology, Binzhou Medical University; Departments of ${ }^{2}$ Gerontology, ${ }^{3}$ Radiology and \\ ${ }^{4}$ Respiratory Medicine, The Affiliated Hospital of Binzhou Medical University, Binzhou, Shandong 256603, P.R. China
}

Received January 17, 2015; Accepted November 24, 2015

DOI: $10.3892 / \mathrm{mmr} .2016 .5118$

\begin{abstract}
Distant metastasis is the predominant pattern of gastric cancer (GC) recurrence, and is the most common cause of cancer-associated mortality. Accumulating evidence has suggested that aberrant activation of epithelial-mesenchymal transition has a crucial role in the genesis, invasion and metastasis of various types of cancer, including GC. Using Cell Counting kit- 8 and Transwell assays, the effects of microRNA (miR)-205 on the proliferation, migration and invasion of NCI-H87 GC cells were determined, and the potential underlying mechanisms were explored. The results of the present study demonstrated that miR-205, which has been reported to function as a tumor suppressor in various types of cancer, significantly suppressed the migration and invasion of GC cells, which may be correlated with its suppressive effects on EMT. Upon transfection with miR-205, the epithelial marker $\mathrm{CDH} 1$ (E-cadherin) was upregulated, and the mesenchymal markers $\mathrm{CDH} 2$ (N-cadherin) and vimentin were suppressed. Furthermore, zinc-finger E-box-binding homeobox factor-1 (ZEB1) was identified as a putative target gene of miR-205 in GC, which may be associated with its suppressive effects. The results of the present study may provide novel diagnostic and therapeutic options for the treatment of human GC.
\end{abstract}

\section{Introduction}

Gastric cancer (GC) is one of the most common types of cancer worldwide. Since the improvement of neoadjuvant treatment for GC, which combines surgery with neoadjuvant chemotherapy, the survival rate of GC has increased; however,

Correspondence to: Dr Xingyue Jiang, Department of Radiology, The Affiliated Hospital of Binzhou Medical University, 661 Huangheer Road, Bincheng, Binzhou, Shandong 256603, P.R. China

E-mail: jxybzmu@163.com

*Contributed equally

Key words: gastric cancer, microRNA-205, epithelial-mesenchymal transition, metastasis in patients with metastatic disease the outcome is worse, with $<30 \%$ survival (1). The etiology of GC is complex, thus resulting in the lack of an internationally accepted standard early prevention regimen (2). Since distant metastasis is the predominant pattern of GC recurrence, and is the most common cause of cancer-associated mortality, it is important to clarify its pathogenesis and to investigate the genes responsible for this progress.

Epithelial-mesenchymal transition (EMT) is an embryonic development program that is associated with changes in cell morphology and increased expression of EMT-associated genes. In cancer, EMT has been reported to confer motility and invasiveness onto cancer cells, leading them to acquire the ability to metastasize to distant sites $(3,4)$. Previous studies have demonstrated that aberrant EMT activation has a crucial role in the genesis, invasion and metastasis of various types of cancer $(5,6)$, including GC (7). Ryu et al (8) analyzed numerous GC specimens and reported that the majority of primary GC tumors, and even premalignant lesions, exhibit a mesenchymal phenotype as characterized by downregulation of $\mathrm{CDHI}$ (E-cadherin), and upregulation of zinc-finger E-box-binding homeobox factor-1 (ZEBI) and SNAII (Snail-1). In addition, $Z E B 1$ and Snail-1 expression levels are positively associated with expression of the cancer stem cell marker CD44 (8). Furthermore, a previous study demonstrated that the expression of $\mathrm{CDH} 2$ (N-cadherin), which is normally expressed in mesenchymal cells, was associated with the invasive phenotype of GC, further suggesting the important role of EMT in the initiation and progression of GC (9).

In addition to traditional transcriptional genes, the role of non-coding microRNAs (miRNAs) on the regulation of EMT has been widely studied (10). miR-205 is a highly conserved miRNA among various species, which has been reported to be closely associated with metastasis in numerous types of cancer $(11,12)$. miR-205 is located in the second intron of the LOC642587 locus in chromosome 1, and has been reported to have an important role in orchestrating the morphogenesis of epithelium during embryogenesis (13). miR-205 has been reported to exhibit consistent overexpression in epidermis (14), whereas, in cells that have undergone EMT progression, its expression is downregulated, alongside a marked downregulation in E-cadherin, and an upregulation in $\mathrm{N}$-cadherin and fibronectin (15). Notably, a previous study reported that by counteracting EMT, miR-205 expression is inversely 
associated with the aggressive behavior of malignant mesothelioma and suppresses its tumor proliferation and invasion (16). These results suggest that miR-205 may act as a suppressor of EMT, as well as a tumor suppressor in cancer. A previous study reported that the expression levels of miR-205 were significantly downregulated in GC tissue, as compared with in normal gastric tissue, and was negatively associated with the clinical and pathological characteristics of patients (17). However, the effects of miR-205 on the metastasis and EMT progression of GC cells, and the underlying molecular mechanisms, remain largely unknown.

The present study aimed to determine whether there was a correlation between miR-205 expression and metastasis of human GC cells. The results demonstrated that restored miR-205 expression resulted in a marked inhibition in the growth, migration and invasion of GC cells. In addition, miR-205 suppressed the EMT progression of GC cells, which may be due to the targeting of the EMT-related transcriptional gene $Z E B 1$.

\section{Materials and methods}

Cell culture and cell transfection. The NCI-H87 human GC cell line was obtained from the American Type Culture Collection (Manassas, VA, USA). The cells were cultured in Dulbecco's modified Eagle medium (DMEM; Gibco; Thermo Fisher Scientific, Inc., Waltham, MA, USA), supplemented with $10 \%$ fetal bovine serum (FBS; PAA Laboratories GmbH, Pasching, Austria), streptomycin (100 $\mu \mathrm{g} / \mathrm{ml}$; Sigma-Aldrich, St. Louis, MO, USA) and penicillin (100 U/ml; Sigma-Aldrich) at $37^{\circ} \mathrm{C}$ in a humidified atmosphere containing 5\% CO 2. miR-205 and scramble mimic were purchased from GE Dharmacon (Lafayette, CO, USA), and were transfected into the cells $\left(1 \times 10^{5}\right)$ at a final concentration of $50 \mathrm{nM}$ using DharmaFECT 1 (GE Dharmacon), according to the manufacturer's protocol. The sequences were as follows: miR-205 5'-GAUUUCAGU GGAGUGAAGUUC-3'; and scramble 5'-UCCUUCAUUCCA CCGGAGUCUG-3'.

Cell Counting kit (CCK)-8 assay. In order to analyze cell proliferation, NCI-H87 cells were seeded into 24-well plates at $5 \times 10^{3}$ cells/well. The cells were incubated in $10 \%$ CCK-8 reagent (Dojindo Molecular Technologies, Inc., Kumamoto, Japan) diluted in normal culture medium at $37^{\circ} \mathrm{C}$, until visual color conversion occurred. The absorbance in each well was measured at 450 and $630 \mathrm{~nm}$ using a microplate reader (Varioskan Flash; Thermo Fisher Scientific, Inc.) at 0, 24, 48 and $72 \mathrm{~h}$ post-transfection.

Cell migration and invasion assays. A Transwell device containing $8 \mu \mathrm{m}$ microporous membranes (Corning, Inc., Corning, NY) was placed into a 24 -well plate. Normal NCI-H87 cells, or NCI-H87 cells $\left(4 \times 10^{5}\right)$ transfected with miR-205 or scramble mimic were seeded in the upper chamber alongside DMEM supplemented with $0.1 \%$ bovine serum albumin (Sigma-Aldrich). DMEM supplemented with $10 \%$ FBS served as chemoattractant. For the invasive assays, the upper and lower chambers of the basal membrane were coated with $5 \mathrm{mg} / \mathrm{ml}$ Matrigel (BD Biosciences, Franklin Lakes, NJ, USA). The rate of migration/invasion was measured after $24 \mathrm{~h}$. The cells adhering to the lower surface were fixed and stained with $0.1 \%$ crystal violet (Sigma-Aldrich), and transferred to a microscope slide. The total number of invading cells was counted in six representative fields under a microscope (BX51; Olympus Corporation, Tokyo, Japan) (magnification, 200x).

RNA extraction and reverse transcription-quantitative polymerase chain reaction $(R T-q P C R)$. Total RNA was extracted from the cells using TRIzol ${ }^{\circledR}$ reagent (Invitrogen; Thermo Fisher Scientific, Inc.), according to the manufacturer's protocol. Total RNA was then reverse transcribed using the First-Strand cDNA Synthesis kit (Invitrogen; Thermo Fisher Scientific, Inc.). The specific primers used for reverse transcription were as follows: miR-205, 5'-TTA TTGCTTAAGAATACGCGTAG-3'; ZEB1, 5'-TTTTTTTTT TTTTTTTTT-3'; and U6, 5'-AAAATATGGAACGCTTCA CGAATTTG-3' (Tsingke Biotechnology, Co., Ltd., Beijing, China). Subsequently, qPCR was performed using the QuantiTect SYBR Green PCR mixture (Invitrogen; Thermo Fisher Scientific, Inc.) on an ABI PRISM 7900 Sequence Detection system (Applied Biosystems; Thermo Fisher Scientific, Inc.). The primers used for qPCR were as follows: miR-205, sense 5'-GCGCTTATTGCTTAAGAATAC-3', anti-sense 5'-CAGTGCAGGGTCCGAGGT-3'; ZEB1, sense 5'-AAACTCGAGTACTTCAATTCCTCGGTATTG-3', anti-sense 5'-AAATCTAGACACACTGTTCTACAGTCC AAGGC-3'; U6, sense 5'-CTCGCTTCGGCAGCACATATA CT-3', anti-sense 5'-ACGCTTCACGAATTTGCGTGTC-3'; and glyceraldehyde 3-phosphate dehydrogenase $(G A P D H)$, sense 5'-TCAACGACCACTTTGTCAAGCTCA-3', and anti-sense 5'-GCTGGTGGTCCAGGGGTCTTACT-3'. The primers were synthesized by Tsingke Biotechnology, Co., Ltd. The expression levels of U6 and GAPDH were used as an internal control for miRNA and mRNA expression, respectively. The PCR cycling conditions were as follows: $94^{\circ} \mathrm{C}$ for $5 \mathrm{sec}$, followed by 40 cycles at $94^{\circ} \mathrm{C}$ for $5 \mathrm{sec}$ and $60^{\circ} \mathrm{C}$ for $34 \mathrm{sec}$, and a final extension step at $72^{\circ} \mathrm{C}$ for $45 \mathrm{sec}$. PCR efficiency was calculated using a relative standard curve derived from a cDNA $(1 \mu \mathrm{l} ; 20 \mathrm{ng} / \mu \mathrm{l})$ mixture, and gave regression coefficients $>0.95$. The relative expression levels were evaluated using the $2^{-\Delta \Delta C q}$ method (18). All experiments were repeated three times, in order to reduce curve-derived variance.

Luciferase reporter assay. The whole 3'-untranslated region (UTR) of ZEB1 was amplified in 293T cells from genomic DNA and cloned into the pGL-3-vector (Promega Corporation, Madison, WI, USA) immediately downstream of the Renilla luciferase gene. A mutated 3'-UTR of ZEB1, in which the miR-205 target site was deleted (Mut), was generated using the QuickChange Site-Directed Mutagenesis kit (Agilent Technologies, Inc., Santa Clara, CA, USA). NCI-H87 cells $\left(1 \times 10^{5} /\right.$ well) were seeded into 24 -well plates $24 \mathrm{~h}$ prior to transfection. The cells were co-transfected with $50 \mathrm{ng}$ pGL-3 firefly luciferase reporter, $10 \mathrm{ng}$ pRL-TK Renilla luciferase reporter and $50 \mathrm{nM}$ miR-205 or scramble mimic using Lipofectamine ${ }^{\circledR} 2000$ (Invitrogen; Thermo Fisher Scientific, Inc.). Cell lysates were prepared using Passive Lysis Buffer (Promega Corporation) $48 \mathrm{~h}$ post-transfection, and luciferase 
activity was measured using the Dual-Luciferase Reporter Assay (Promega Corporation). Results were normalized to Renilla luciferase.

Rescue assay. The full length ZEBI gene open reading frame (ORF) was amplified by PCR and cloned into a pCDNA-3.1 construct (Promega Corporation), in order to generate the pCDNA-3.1-ZEB1 construct. Briefly, ZEB1 ORF was extracted for EcoRV and Xbal double digestion (New England Biolabs, Inc., Ipswich, MA, USA), and purified gene fragments were recovered. EcoRV and $\mathrm{Xbal}$ double digestion of the pcDNA-3.1 expression vector was then performed. The recovered target gene fragments were ligated into digested pcDNA-3.1 expression vectors and identified using agarose gel electrophoresis. An empty pCDNA-3.1 construct was used as the control. The NCI-H87 cells were initially transfected with miR-205 or scramble mimic $(60 \mathrm{nM})$ in 6-well plates. Following a $24 \mathrm{~h}$ culture, the NCI-H87 cells were co-transfected with miR-205 mimic $(30 \mathrm{nM})$ and $2.0 \mu \mathrm{g}$ of either pcDNA-3.1-ZEB1 or pcDNA-3.1 constructs. The cells were harvested at predetermined intervals and assessed as necessary.

Western blot analysis. For western blotting, the cells were harvested in ice-cold phosphate-buffered saline $48 \mathrm{~h}$ post-transfection, and lysed on ice in cold modified radioimmunoprecipitation buffer (Beyotime Institute of Biotechnology, Haimen, China) supplemented with protease inhibitors (Roche Diagnostics, Basel, Switzerland). Protein concentration was determined using the Bicinchoninic Acid Protein Assay kit (Vigorous Biotechnology Beijing Co., Ltd., Beijing, China) and equal amounts of protein $(30 \mu \mathrm{g})$ were separated by $10 \%$ sodium dodecyl sulfate-polyacrylamide gel electrophoresis. The gels were electroblotted onto nitrocellulose membranes (EMD Millipore, Billerica, MA, USA). The membranes were subsequently blocked for $2 \mathrm{~h}$ with 5\% non-fat dry milk in Tris-buffered saline containing $0.1 \%$ Tween-20, and incubated at $4^{\circ} \mathrm{C}$ overnight with primary antibody. Detection was performed using alkaline phosphatase-conjugated anti-mouse (cat. no. 7056) and anti-rabbit (cat. no. 7054) immunoglobulin G secondary antibodies $(1: 5,000)$ and the blots were visualized using an enhanced chemiluminescence system (EMD Millipore). The results of western blotting were analyzed using Quantity One v4.6.2 (Bio-Rad Laboratories, Inc., Hercules, CA, USA). The primary antibodies used were as follows: Rabbit monoclonal anti-human ZEB (1:1,000; cat. no. 3396), mouse monoclonal anti-human E-cadherin (1:1,000; cat. no. 14472), rabbit monoclonal anti-human vimentin $(1: 1,000$; cat. no. 5741), rabbit monoclonal anti-human N-cadherin (1:1,000; cat. no. 13116) and rabbit monoclonal anti-human GAPDH (1:10,000; cat. no. 5174), which was used as a negative control. All primary and secondary antibodies were purchased from Cell Signaling Technology, Inc. (Danvers, MA, USA).

Statistical analysis. All experiments were repeated at least three times. Data are presented as the mean \pm standard deviation of repeated experiments. Statistical analysis was carried out using SPSS 15.0 software (SPSS Inc., Chicago, IL, USA). Two-tailed Student's t-test was used to analyze the data.
$\mathrm{P}<0.05$ was considered to indicate a statistically significant difference.

\section{Results}

miR-205 suppresses the proliferation, migration and invasion of NCI-H87 cells. A previous study reported a downregulation of miR-205 in GC tissue samples (17); however, its biological significance on cancer progression remains unclear. To further explore the effects of miR-205 on the malignant phenotype of GC cells, NCI-H87 cells in which miR-205 was underexpressed (17), were exogenously transfected with miR-205 or a scramble mimic. Upon transfection, the intracellular expression levels of miR-205 were $\sim 100$-fold higher the NCI-H87 cells transfected with the miR-205 mimic, as compared with the scramble control group (Fig. 1A). In addition, the effects of miR-205 on cell proliferation were determined using a CCK-8 assay. As shown in Fig. 1B, treatment with miR-205 significantly suppressed the cell growth rate of the NCI-H87 cells.

Since miR-205 is closely associated with tumor metastasis, the present study hypothesized that miR-205 may have an important role in GC cell migration and invasion, which promote tumor metastasis, giving rise to $\mathrm{GC}$-associated mortality. Therefore, the present study explored the effects of miR-205 on the migration and invasion of NCI-H87 cells using a Transwell assay. A Transwell assay without Matrigel demonstrated that overexpression of miR-205 in NCI-H87 cells resulted in a significant reduction in the number of cells that passed through the chambers, as compared with the scramble control group $(240 \pm 45$ vs. $85 \pm 15$ cells) $(\mathrm{P}<0.05$; Fig. $1 \mathrm{C})$. Subsequently, the chambers were coated with Matrigel, which mimics the extracellular matrix, prior to experimentation. The invasion assay exhibited similar results to the migration assay. As shown in Fig. 1D, miR-205 overexpression significantly reduced the number of NCI-H87 cells that passed through the chambers $(104 \pm 26$ vs. $42 \pm 18$ cells $)(P<0.05)$. These results indicate that miR-205 may efficiently suppress the motility and invasiveness of GC cells in vitro.

miR-205 promotes an epithelial phenotype in GC cells. Since miR-205 can inhibit gastric cancer cell migration and invasion, the present study hypothesized that it may be associated with the inhibition of EMT progression in GC cells. Since EMT is often associated with a decrease or loss of epithelial markers, including E-cadherin, and a gain of mesenchymal markers, including vimentin and $\mathrm{N}$-cadherin, the present study detected the molecular alterations in cells overexpressing miR-205. The protein expression levels of mesenchymal and epithelial markers were detected in the NCI-H87 cells. As shown in Fig. 2, the mesenchymal markers vimentin and $\mathrm{N}$-cadherin were consistently suppressed in NCI-H87 cells treated with the miR-205 mimic. However, the epithelial marker E-cadherin was markedly upregulated following transfection with miR-205. These results suggest that overexpression of miR-205 may induce an epithelial phenotype in GC cells.

ZEB1 is a putative target gene of miR-205 in GC cells. To explore the target genes associated with GC tumor progression triggered by miR-205, putative targets of miR-205 were searched using prediction programs (Targetscan, http://genes. 
A

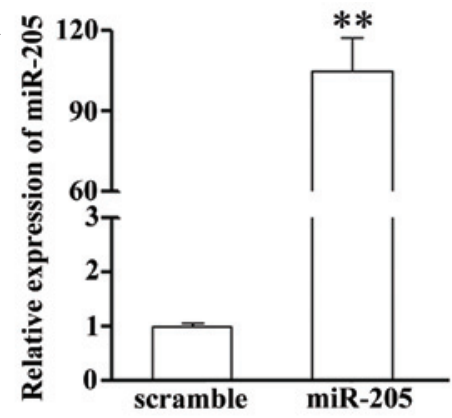

C

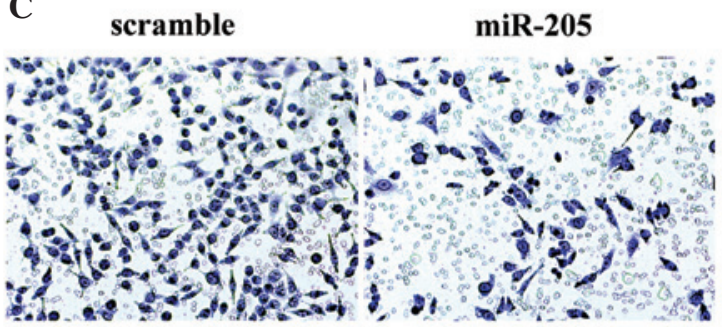

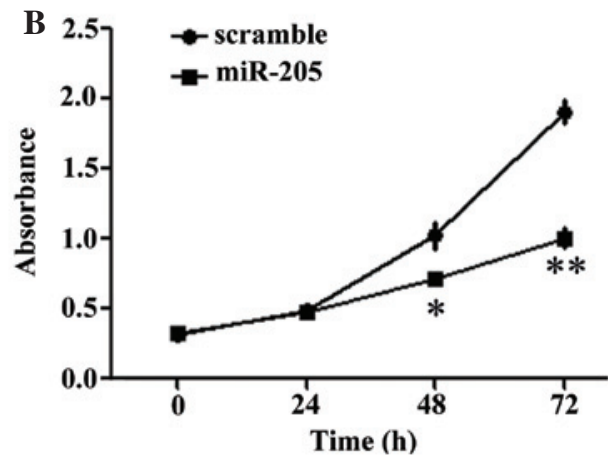

D

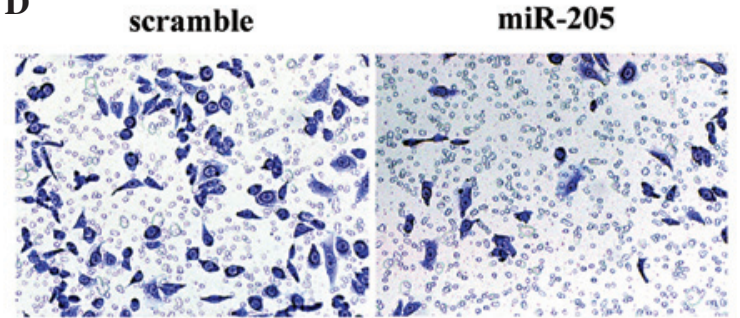

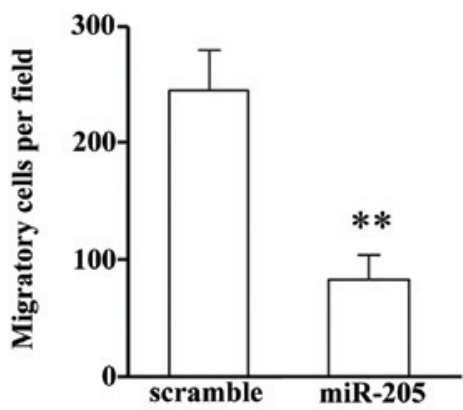

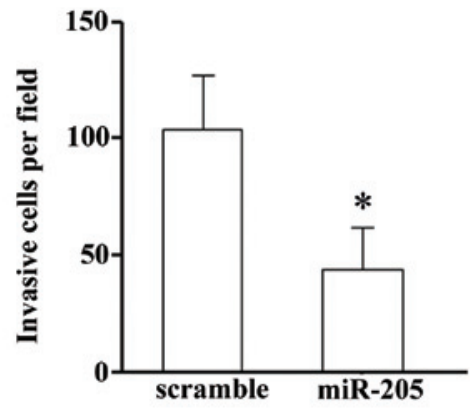

Figure 1. Effects of mircoRNA (miR)-205 on NCI-H87 gastric cancer cell proliferation and invasion. (A) Expression levels of miR-205 were detected in NCI-H87 cells following transfection with miR-205 or a scramble mimic by quantitative polymerase chain reaction. (B) Cell proliferation of NCI-H87 cells following transfection with miR-205 or a scramble mimic, as detected using Cell Counting kit-8. (C) Cell migration of NCI-H87 cells following transfection with miR-205 or a scramble mimic, as detected using a Transwell assay. The relative number of cells that passed through the membrane per field is shown. (D) Cell invasion of NCI-H87 cells following transfection with miR-205 or a scramble mimic, as detected using a Transwell assay. The relative ratio of invasive cells per field is shown. Data are presented as the mean \pm standard deviation. ${ }^{*} \mathrm{P}<0.05,{ }^{* *} \mathrm{P}<0.01$ vs. the scramble group.

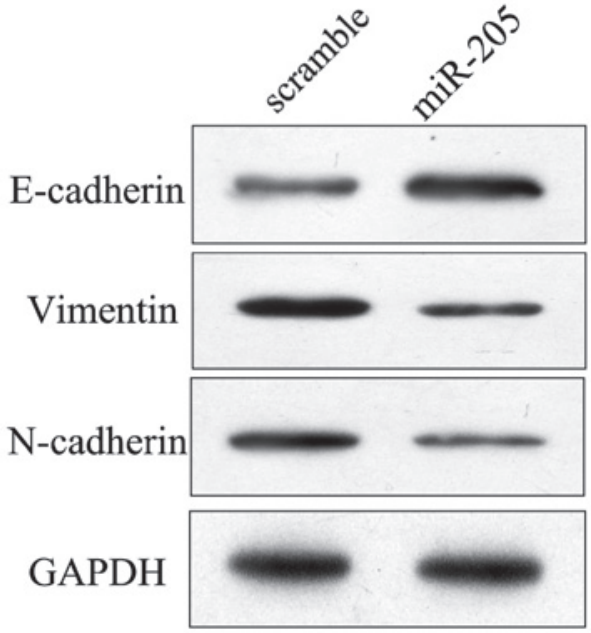

Figure 2. MicroRNA (miR)-205 induced an epithelial phenotype in NCI-H87 gastric cancer cells. Western blot analysis was used to determine the expression of epithelial and mesenchymal marker proteins in NCI-H87 cells transfected with miR-205 or a scramble mimic. Following transfection, the mesenchymal markers vimentin and $\mathrm{N}$-cadherin were suppressed, whereas the epithelial marker E-cadherin was upregulated. GAPDH, glyceraldehyde 3-phosphate dehydrogenase. mit.edu/targetscan/ and miRanda, http://www.microrna.org/ microrna/home.do). Among the common predicted targets of miR-205, ZEBI was selected as an ideal candidate due to its important role in EMT (15). ZEB1 has previously been reported as a target of miR-205 in breast cancer (19); however, the interaction between miR-205 and ZEB-1 has not been experimentally validated in GC.

To confirm miR-205 binding within the 3'-UTR of ZEB1, a mutated 3'-UTR of ZEB1, in which the miR-205 target site was deleted, was generated (Fig. 3A). Subsequently, the effects of Mut and wild type 3'UTR constructs on NCI-H87 cells overexpressing miR-205 were determined using a dual-luciferase detection system. As a result, significant suppression of luciferase activities were observed in the NCI-H87 cells co-transfected with the wild type 3'UTR construct and miR-205 mimic, as compared with the Mut construct groups (Fig. 3B). These results suggest that miR-205 may suppress the transcriptional activity of the ZEBI gene by targeting the binding site in the 3'UTR of ZEB1 mRNA. Consistent with the reporter assays, transfection with miR-205 decreased the mRNA expression levels of $Z E B I$, as compared 
A

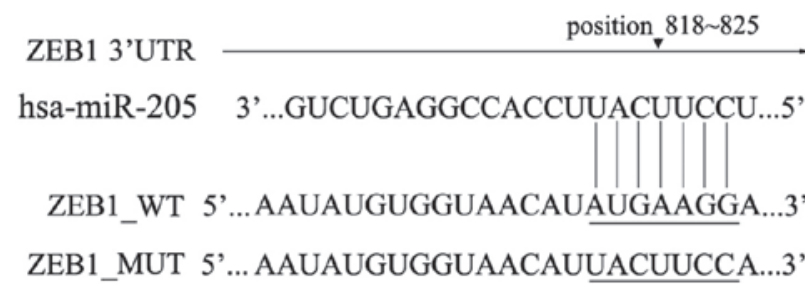

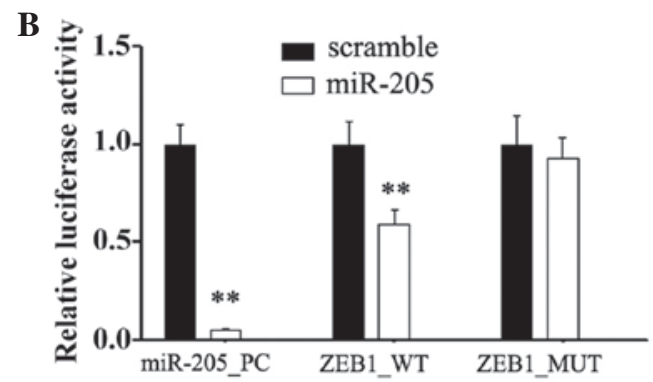

D

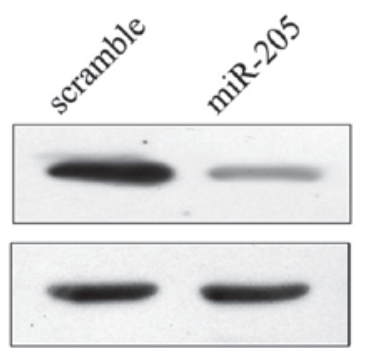

Figure 3. MicroRNA (miR)-205 targets zinc-finger E-box-binding homeobox factor-1 (ZEB1) gene in NCI-H87 gastric cancer cells. (A) Schematic representation of ZEB1 3'-untranslated region (UTR) showing a putative miR-205 target site. (B) Relative luciferase activity of the indicated ZEB1 reporter construct in NCI-H87 cells co-transfected with miR-205 or a scramble mimic. (C) Quantitative polymerase chain reaction was performed to detect the expression levels of ZEB1 following trasnfection with miR-205 or a scramble mimic. (D) Western blot analysis of the protein expression levels of ZEB1 in NCI-H87 cells transfected with miR-205 or a scramble mimic was performed. Data are presented as the mean \pm standard deviation. ${ }^{* *} \mathrm{P}<0.01$ vs. the scramble group. PC, pcDNA-3.1 control; WT, wild type; MUT, mutant; GAPDH, glyceraldehyde 3-phosphate dehydrogenase.

with in the scramble group (Fig. 3C). In addition, according to immunoblotting results, changes were detected in the protein expression levels of ZEB1 post-transfection with a miR-205 mimic. These results indicate that miR-205 may directly target the expression of $Z E B I$ in GC cells.

ZEB1 is associated with the miR-205-mediated suppression of proliferation and EMT progression. ZEBI is a transcriptional inducer of EMT in cancer of epithelial origin, and has been reported to have a key role in tumor metastasis (20). However, whether it is involved in miR-205-mediated suppression of migration and EMT progression in NCI-H87 cells remains unclear. Therefore, a 'rescue' methodology was adopted to examine the functional relevance of miR-205-ZEBI interaction in NCI-H87 cells. A novel construct containing the full ORF of ZEB1 was generated. Subsequently, NCI-H87 cells were co-transfected with miR-205 or a scramble mimic alongside pcDNA-3.1-ZEB1 or pcDNA-3.1 control constructs. Post-transfection, the expression levels of $Z E B 1$ were restored when the ZEBI construct was transfected into the NCI-H87 cells that had been transfected with a miR-205 mimic for $24 \mathrm{~h}$ (Fig. 4A). In agreement with the restored expression of $Z E B 1$, increased cell proliferation was observed in the NCI-H87 cells transfected with the ZEB1 construct following transfection with the miR-205 mimic (Fig. 4B). Furthermore, post-transfection with the $Z E B 1$ construct, the miR-205-mediated suppression of cell invasion (Fig. 4C) in NCI-H87 cells was also partially attenuated. The number of invasive cells in the pcDNA-ZEB1 + miR-205 and scramble + pcDNA3.1 groups were significantly reduced, as compared with in the scramble + pcDNA-ZEBlgroup, and were significantly increased as compared with in the miR-205 + pcDNA3.1 group. Consistent with the restored expression of $Z E B 1$, suppression of $\mathrm{N}$-cadherin was restored, and the upregulation of E-cadherin was partially attenuated (Fig. 4A). These results indicate that ZEBl may be a functional target of miR-205, contributing to its role in the miR-205-mediated suppression of cell invasion and EMT progression in GC cells.

\section{Discussion}

EMT is a fundamental process in embryonic development, which is also considered an important step leading to tumor invasion and metastasis (21). At present, previously unknown markers, miRNAs, are considered to be important components of the cancer signaling network and are emerging as novel biomarkers of numerous diseases (22). miRNAs are a group of endogenous, small, non-coding RNAs that modulate protein expression by regulating the translational efficiency or cleavage of targets (23). By partially complementing the 3'-UTR of specific mRNAs, miRNAs induce the genetic silencing of various target mRNAs, which are involved in numerous biological processes, including EMT regulation (24). Previous studies have reported the important role of miRNAs in the initiation and progression of GC (25-27), and numerous tumor-associated circulating miRNAs have been reported to possess potential as novel, non-invasive biomarkers for the early detection of GC (28). Therefore, improved knowledge regarding alterations in miRNA expression during GC progression and metastasis may provide novel options for the diagnosis and treatment of GC. The present study provided important evidence in support of miR-205 functioning as a tumor suppressor in GC.

miR-205 is a highly conserved gene among various species, which has been closely associated with metastasis in numerous types of cancer $(11,12)$. In melanoma specimens, E2F transcription factor $1(E 2 F 1)$ is negatively regulated by miR-205. Overexpression of miR-205 leads to a mediation of E2F1-regulated Akt phosphorylation and an upregulation of 


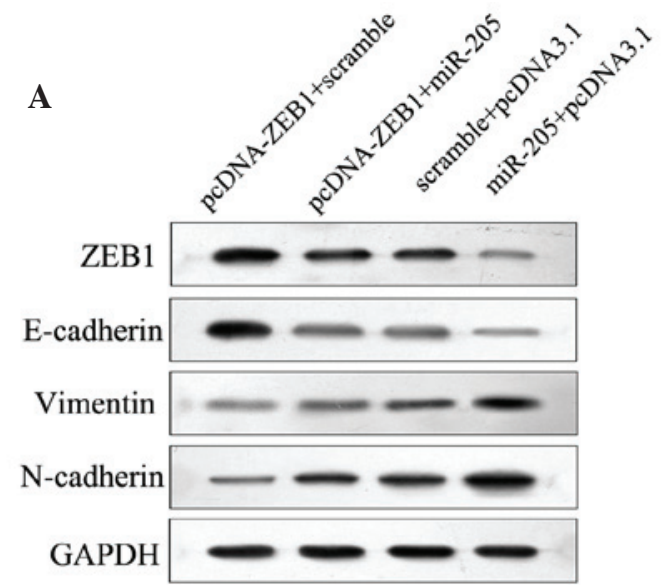

C

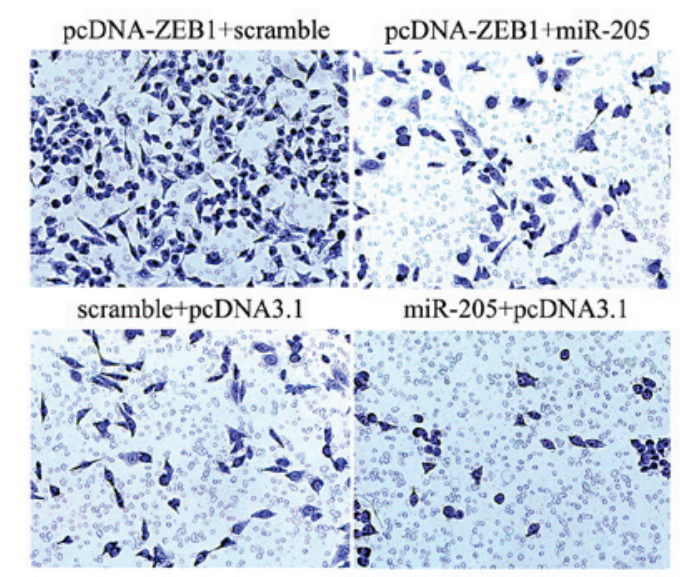

B
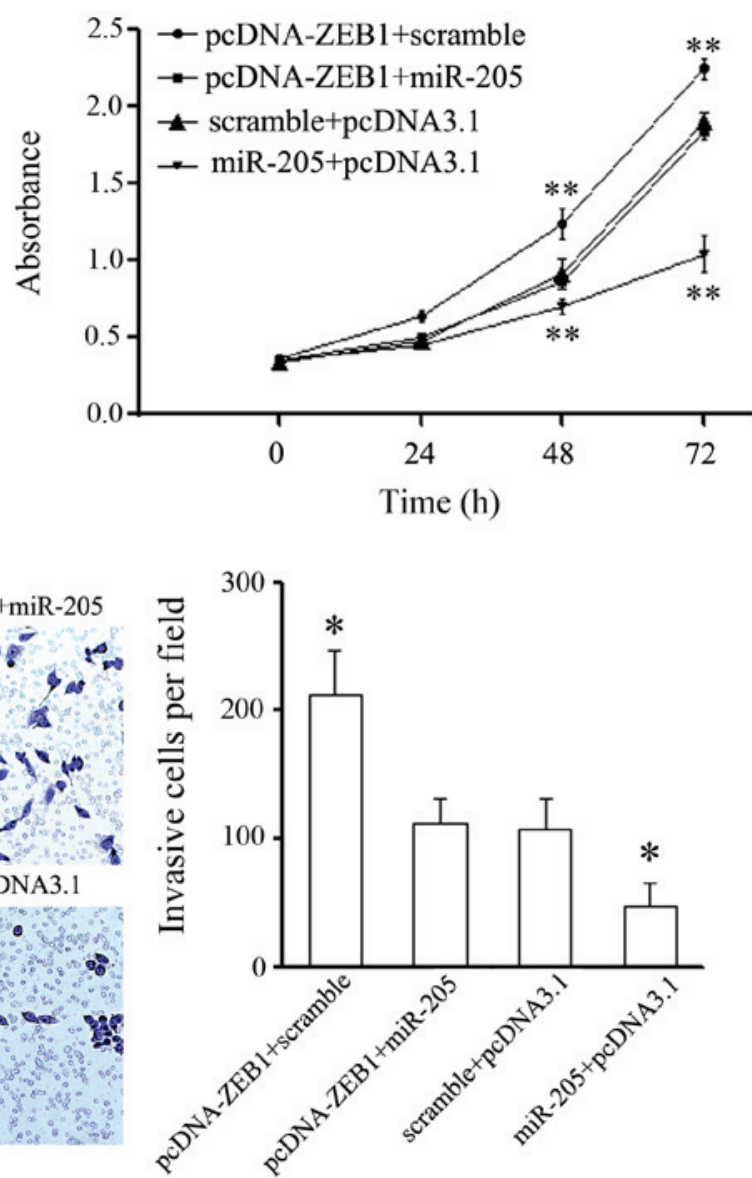

Figure 4. Zinc-finger E-box-binding homeobox factor-1 (ZEB1) is involved in microRNA (miR)-205-dependent control of NCI-H87 gastric cancer cell proliferation and invasion. (A) Protein expression levels of ZEB1, vimentin, N-cadherin and E-cadherin in NCI-H87 cells co-transfected with either miR-205 or a scramble mimic, and pcDNA-3.1-ZEB1 or pcDNA-3.1 empty vector, as detected by western blotting. (B) Cell Counting kit-8 assays were performed to detect the effects of ZEB1 on miR-205-mediated cell proliferation inhibition. ${ }^{* *} \mathrm{P}<0.05$ vs. pcDNA-ZEB1 + miR-205 and scramble + pcDNA3.1. (C) Cell invasion assays of NCI-H87 cells were performed to detect the effects of $Z E B 1$ on miR-205-mediated cell invasion inhibition. ${ }^{*} \mathrm{P}<0.01 \mathrm{vs}$. pcDNA-ZEB1 + miR-205 and scramble + pcDNA3.1. Data are presented as the mean \pm standard deviation.

p16-INK4A, resulting in the suppression of cell proliferation (29). Furthermore, by counteracting EMT, miR-205 expression is inversely associated with the aggressive behavior of malignant mesothelioma and is able to suppress its tumor proliferation and invasion (16). A previous study reported that the expression levels of miR-205 were significantly downregulated in GC tissue, as compared with in normal gastric tissue, and was negatively associated with the clinical and pathological characteristics of patients (17). In addition, inhibition of miR-205 significantly promoted the proliferation of GC cells, thus suggesting the suppressive role of miR-205 in GC. However, the effects of miR-205 on metastasis and EMT progression of GC cells, and the underlying molecular mechanisms, remain largely unknown. The present study demonstrated that restored expression of miR-205 in the NCI-H87 GC cell line resulted in inhibition of cell proliferation, migration and invasion. Alongside suppressed cell invasion, miR-205 suppressed the expression of epithelial markers and upregulated the expression of mesenchymal markers in GC cells. The present study further confirmed the effects of miR-205 on the biological function of GC cells, thus suggesting the tumor suppressor role of miR-205 in GC.
To further identify the mechanisms underlying the suppressive effects of miR-205, the putative targets of miR-205 were explored. Among these genes, ZEBl was selected. ZEB1, which is a member of the ZEB family, is a transcriptional repressor that mediates its binding to paired CAGGTA/G E-box-like promoter elements (30). Through suppressing the expression of E-cadherin $Z E B 1$ induces EMT and contributes to the progression of malignant cancer (31). In addition, previous studies have demonstrated that $Z E B 1$ expression is positively correlated with drug resistance in cancer cells (19), and ZEBI knockdown was able to chemosensitize pancreatic cancer cells to conventional chemotherapy drugs, including gemcitabine, 5-fluorouracil and cisplatin (32). The results of the present study demonstrated that alterations in miR-205 expression in GC cells led to the opposite effects associated with $Z E B 1$ alterations, highlighting its negative regulation. Furthermore, the ZEB1 mRNA 3'-UTR bears a binding site of miR-205, and transfection with miR-205 resulted in the suppression of the mRNA and protein expression levels of $Z E B 1$.

To further analyze whether $Z E B 1$ has an important role in miR-205-mediated suppression of cell proliferation 
and invasion, further rescue assays were performed. It was suggested that miR-205 inhibited EMT progression via targeting ZEBI in GC cells, since restored expression of ZEBI could partially attenuate miR-205-mediated down-regulation of $\mathrm{N}$-cadherin and up-regulation of E-cadherin. Consistent with the suppression of EMT, the suppression of proliferation and invasion were also partially restored. However, overexpression of ZEBI could not completely abolish miR-205-mediated tumor suppression, thus suggesting that other target genes may be involved in the suppression of GC. Therefore, further research is required, in order to elucidate the exact mechanisms underlying miR-205-mediated functions in GC cells. As more information regarding the mechanisms is obtained, the opportunity to manipulate them in cancer in order to suppress tumor metastasis may arise.

In conclusion, the results of the present study indicated that aberrant expression of miR-205 may have a role in the tumor progression and prognosis of patients with GC. Furthermore, these data suggested that miR-205 may function as a tumor suppressor, and may modulate GC cell proliferation, invasion and EMT progression by directly and negatively regulating ZEB1. Therefore, the restored expression of miR-205 may be considered a potential therapeutic strategy for the treatment of GC.

\section{Acknowledgements}

The present study was supported by funds provided by the Science and Technology Plan of Shandong University (grant no. J11LF66).

\section{References}

1. Gill RS, Al-Adra DP, Nagendran J, Campbell S, Shi X, Haase E and Schiller D: Treatment of gastric cancer with peritoneal carcinomatosis by cytoreductive surgery and HIPEC: A systematic review of survival, mortality, and morbidity. J Surg Oncol 104: 692-698, 2011

2. Kanat $\mathrm{O}$ and $\mathrm{O}^{\prime} \mathrm{Neil} \mathrm{BH}$ : Metastatic gastric cancer treatment: A little slow but worthy progress. Med Oncol 30: 464, 2013.

3. Christiansen JJ and Rajasekaran AK: Reassessing epithelial to mesenchymal transition as a prerequisite for carcinoma invasion and metastasis. Cancer Res 66: 8319-8326, 2006.

4. Klymkowsky MW and Savagner P: Epithelial-mesenchymal transition: A cancer researcher's conceptual friend and foe. Am J Pathol 174: 1588-1593, 2009.

5. Montemayor-Garcia C, Hardin H, Guo Z, Larrain C, Buehler D, Asioli S, Chen H and Lloyd RV: The role of epithelial mesenchymal transition markers in thyroid carcinoma progression. Endocr Pathol 24: 206-212, 2013.

6. Liang Q, Li L, Zhang J, Lei Y, Wang L, Liu DX, Feng J, Hou P, Yao R, Zhang Y, et al: CDK5 is essential for TGF- $\beta 1$-induced epithelial-mesenchymal transition and breast cancer progression. Sci Rep 3: 2932, 2013.

7. Zhao L, Li W, Zang W, Liu Z, Xu X, Yu H, Yang Q and Jia J: JMJD2B promotes epithelial-mesenchymal transition by cooperating with $\beta$-catenin and enhances gastric cancer metastasis. Clin Cancer Res 19: 6419-6429, 2013.

8. Ryu HS, Park do J, Kim HH, Kim WH and Lee HS: Combination of epithelial-mesenchymal transition and cancer stem cell-like phenotypes has independent prognostic value in gastric cancer. Hum Pathol 43: 520-528, 2012.

9. Hazan RB, Qiao R, Keren R, Badano I and Suyama K: Cadherin switch in tumor progression. Ann NY Acad Sci 1014: 155-163, 2004.

10. Garg M: Targeting microRNAs in epithelial-to-mesenchymal transition-induced cancer stem cells: Therapeutic approaches in cancer. Expert Opin Ther Targets 19: 285-297, 2015.
11. Kalogirou C, Spahn M, Krebs M, Joniau S, Lerut E, Burger M, Scholz CJ, Kneitz S, Riedmiller H and Kneitz B: MiR-205 is progressively down-regulated in lymph node metastasis but fails as a prognostic biomarker in high-risk prostate cancer. Int J Mol Sci 14: 21414-21434, 2013.

12. Tucci P, Agostini M, Grespi F, Markert EK, Terrinoni A, Vousden KH, Muller PA, Dötsch V, Kehrloesser S, Sayan BS, et al: Loss of p63 and its microRNA-205 target results in enhanced cell migration and metastasis in prostate cancer. Proc Natl Acad Sci USA 109: 15312-15317, 2012

13. Yi R, O'Carroll D, Pasolli HA,ZZhang Z, Dietrich FS, Tarakhovsky A and Fuchs E: Morphogenesis in skin is governed by discrete sets of differentially expressed microRNAs. Nat Genet 38: 356-362, 2006.

14. Ason B, Darnell DK, Wittbrodt B, Berezikov E, Kloosterman WP, Wittbrodt J, Antin PB and Plasterk RH: Differences in vertebrate microRNA expression. Proc Natl Acad Sci USA 103: 14385-14389, 2006.

15. Gregory PA, Bert AG, Paterson EL, Barry SC, Tsykin A, Farshid G, Vadas MA, Khew-Goodall Y and Goodall GJ: The miR-200 family and miR-205 regulate epithelial to mesenchymal transition by targeting ZEB1 and SIP1. Nat Cell Biol 10: 593-601, 2008.

16. Fassina A, Cappellesso R, Guzzardo V, Dalla Via L, Piccolo S, Ventura L and Fassan M: Epithelial-mesenchymal transition in malignant mesothelioma. Mod Pathol 25: 86-99, 2012.

17. Yin WZ, Li F, Zhang L, Ren XP, Zhang N and Wen JF: Down-regulation of microRNA-205 promotes gastric cancer cell proliferation. Eur Rev Med Pharmacol Sci 18: 1027-1032, 2014.

18. Schmittgen TD, Zakrajsek BA, Mills AG, Gorn V, Singer MJ and Reed MW: Quantitative reverse transcription-polymerase chain reaction to study mRNA decay: Comparison of endpoint and real-time methods. Anal Biochem 285: 194-204, 2000.

19. Lee JY, Park MK, Park JH, Lee HJ, Shin DH, Kang Y, Lee CH and Kong G: Loss of the polycomb protein Mel-18 enhances the epithelial-mesenchymal transition by ZEB1 and ZEB2 expression through the downregulation of miR-205 in breast cancer. Oncogene 33: 1325-1335, 2014.

20. Wellner U, Schubert J, Burk UC, Schmalhofer O, Zhu F, Sonntag A, Waldvogel B, Vannier C, Darling D, zur Hausen A, et al: The EMT-activator ZEB1 promotes tumorigenicity by repressing stemness-inhibiting microRNAs. Nat Cell Biol 11: 1487-1495, 2009.

21. Thiery JP and Sleeman JP: Complex networks orchestrate epithelial-mesenchymal transitions. Nat Rev Mol Cell Biol 7: 131-142, 2006.

22. Kong YW, Ferland-McCollough D, Jackson TJ and Bushell M: microRNAs in cancer management. Lancet Oncol 13: e249-e258, 2012.

23. Bartel DP: MicroRNAs: Genomics, biogenesis, mechanism and function. Cell 116: 281-297, 2004.

24. Chen K and Rajewsky N: The evolution of gene regulation by transcription factors and microRNAs. Nat Rev Genet 8: 93-103, 2007.

25. Hui A, How C, Ito E and Liu FF: Micro-RNAs as diagnostic or prognostic markers in human epithelial malignancies. BMC Cancer 11: $500,2011$.

26. Yanaka Y, Muramatsu T, Uetake H, Kozaki $\mathrm{K}$ and Inazawa J: miR-544a induces epithelial-mesenchymal transition through the activation of WNT signaling pathway in gastric cancer Carcinogenesis 36: 1363-1371, 2015.

27. Zhang X, Peng Y, Jin Z, Huang Q, Cheng Y, Liu Y, Feng X, Yang M, Huang Y, Zhao Z, et al: Integrated miRNA profiling and bioinformatics analyses reveal potential causative miRNAs in gastric adenocarcinoma. Oncotarget 6: 32878-32889, 2015.

28. Blanco-Calvo M, Calvo L, Figueroa A, Haz-Conde M, Anton-Aparicio L and Valladares-Ayerbes M: Circulating microRNAs: Molecular microsensors in gastrointestinal cancer. Sensors (Basel) 12: 9349-9362, 2012.

29. Dar AA, Majid S, de Semir D, Nosrati M, Bezrookove V and Kashani-Sabet M: miRNA-205 suppresses melanoma cell proliferation and induces senescence via regulation of E2F1 protein. J Biol Chem 286: 16606-16614, 2011.

30. Brabletz S and Brabletz T: The ZEB/miR-200 feedback loop - a motor of cellular plasticity in development and cancer? EMBO Rep 11: 670-677, 2010.

31. Gheldof A, Hulpiau P, van Roy F, De Craene B and Berx G: Evolutionary functional analysis and molecular regulation of the ZEB transcription factors. Cell Mol Life Sci 69: 2527-2541, 2012.

32. Arumugam T, Ramachandran V, Fournier KF, Wang $\mathrm{H}$, Marquis L, Abbruzzese JL, Gallick GE, Logsdon CD, McConkey DJ and Choi W: Epithelial to mesenchymal transition contributes to drug resistance in pancreatic cancer. Cancer Res 69: 5820-5828, 2009 . 